\title{
A classification of methods used to attach devices to vultures and condors
}

\section{Mary Davies \& Rhys Green}

There are many publications about the results obtained from deploying tracking and telemetry devices on vultures and condors, but only a few provide precise details of the design of the attachment apparatus used. Frequently, reference is made to a generic publication about the type of attachment method used, such as the much-cited publications by Kenward (2001) and Rappole \& Tipton (1991) about particular designs, but enquiries we have made to authors often reveal that they made several modifications, or even used a completely different design from that described in the cited publication. Hence, we suggest that there are actually more types of attachment than one might think from reading the scientific literature. For this reason, we asked all expert participants in the technical workshop held at the International Centre for Birds of Prey at Newent, UK, on 20-22 August 2019, to contribute information on attachment methods they had used or knew about. These were summarised in the series of diagrams, showing 12 different attachment types, presented below. We do not know of any widely accepted classification or terminology for attachment designs. Terms such as "backpack" and "wing-loop harness" are often used in Methods texts, but http://dx.doi.org/10.4314/vulnew.v78ai1.4

authors frequently mean quite different things by them. We suggest some names for different attachment designs below and propose them as a first step towards a standardised terminology. We would welcome corrections, revisions and additions to this prototype. We do not intend, by suggesting this classification, to endorse or approve any of these designs; only to define what they are and what to call them.

Although our classification identified 12 types of attachment method, the experts present at the Global Workshop on Methods for Attaching Tracking Devices to Vultures and Condors demonstrated just three of these broad classes; the thoracic X-strap harness, the leg loop harness and the patagial mount. However, we were struck by the differences between groups of researchers in the details of the construction and fitting of harnesses of the same broad type. Hence, the following practical step-by-step guides describe three variants of the thoracic X-strap harness and three variants of the leg loop harness and one version of the patagial mount.

Figure B.1 shows diagrams of 12 attachment designs used for research devices deployed on Vultures and Condors. 


\section{Lateral}

1.

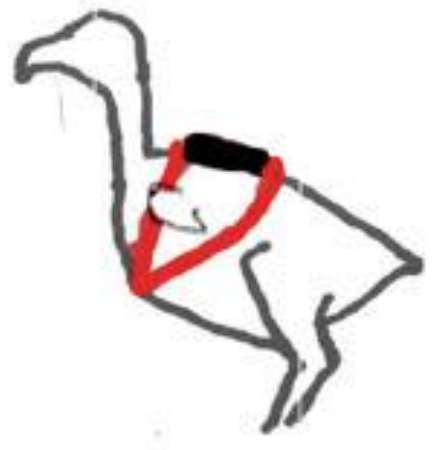

Thoracic (X-strap with forward strap)

3.
Ventral

Thoracic (X-strap)
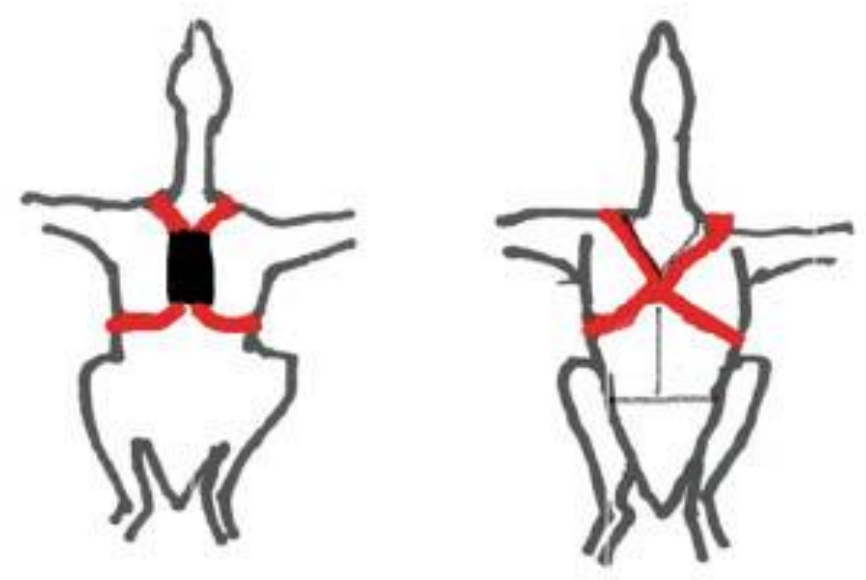

Thoracic (X-strap with back strap)
2.
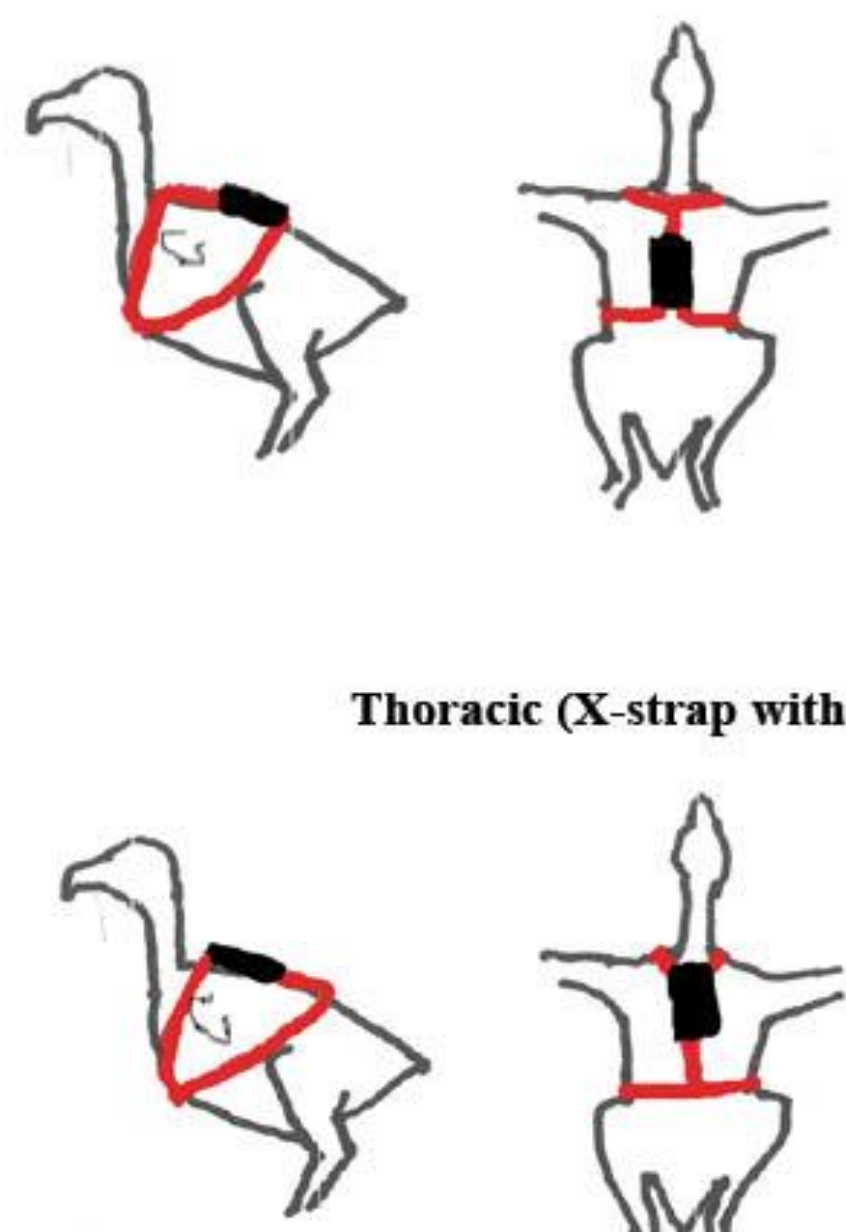
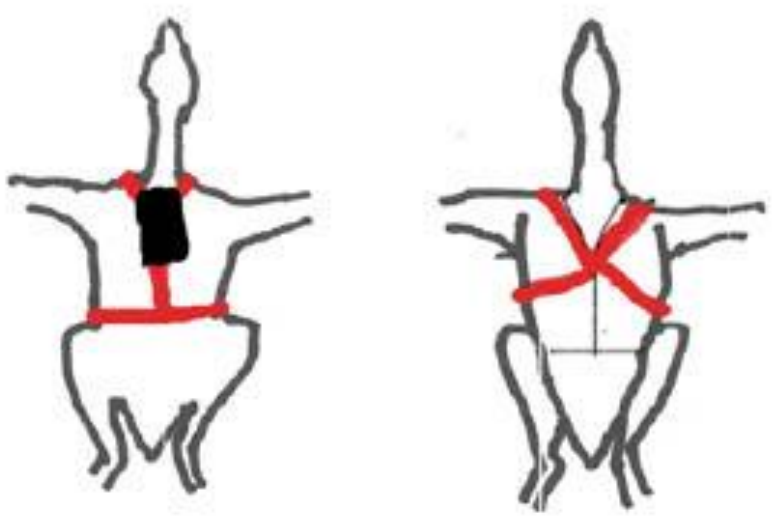


\section{Lateral}

4.

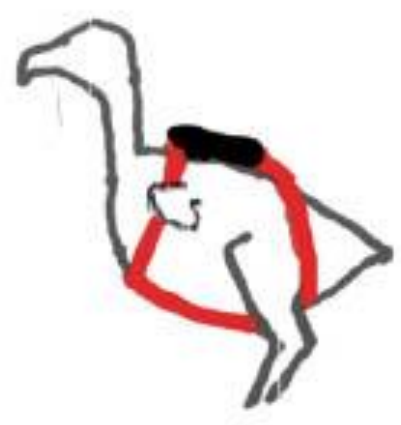

5.

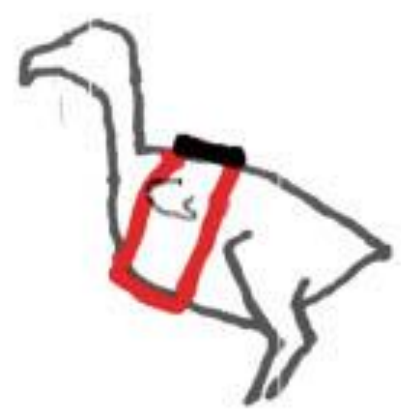

Dorsal

Thoracic (parachute)
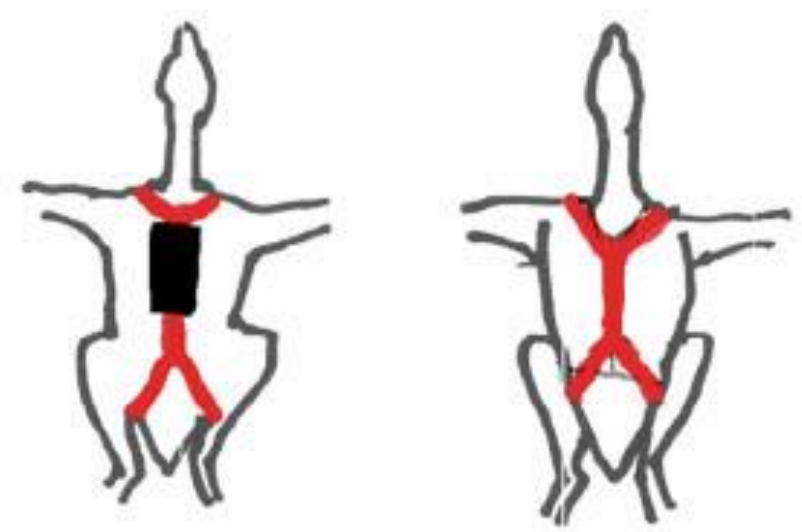

Thoracic (breast strap)
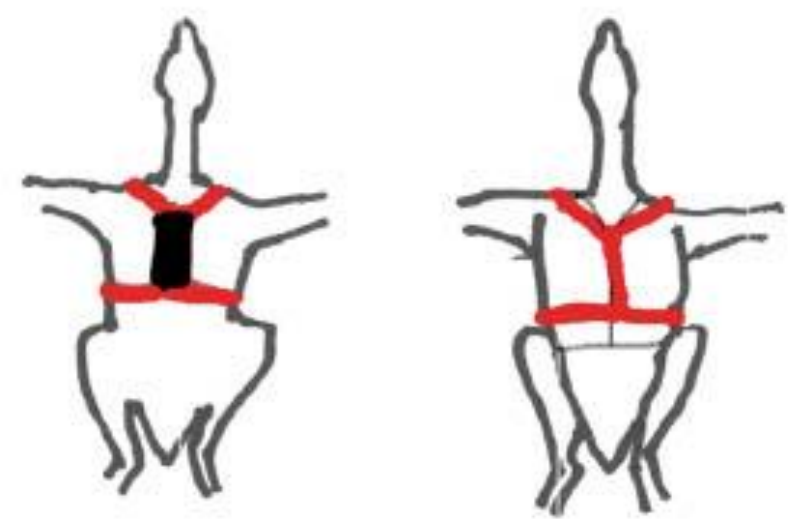

Ventral
6.

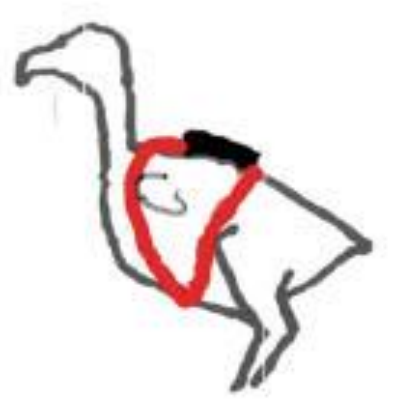




\section{Lateral}

7.

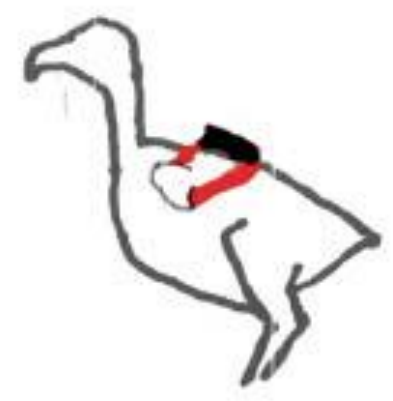

Dorsal

Wing loop

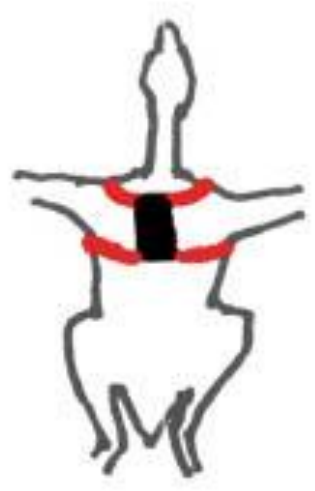

Ventral

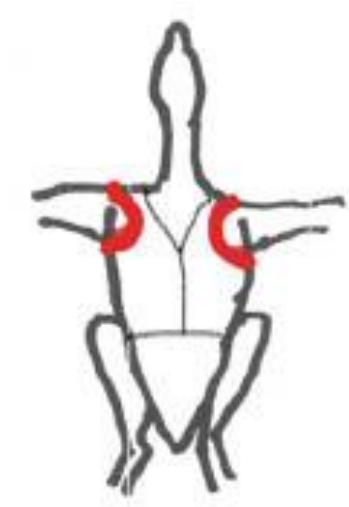

8.

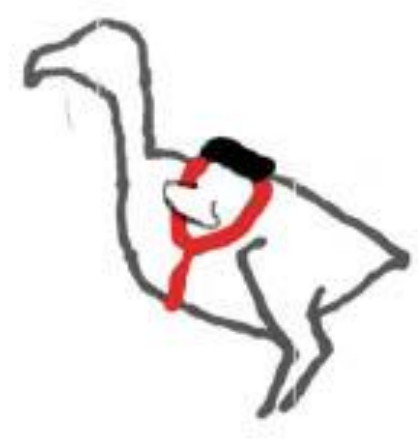

9.

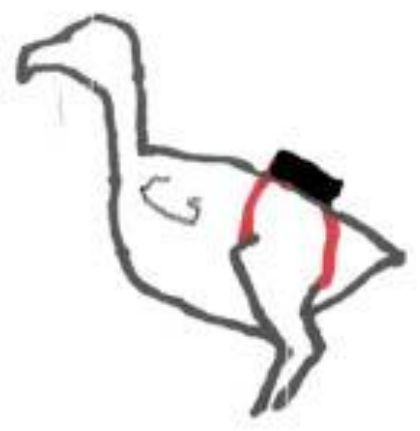

\section{Wing loop (breast strap)}
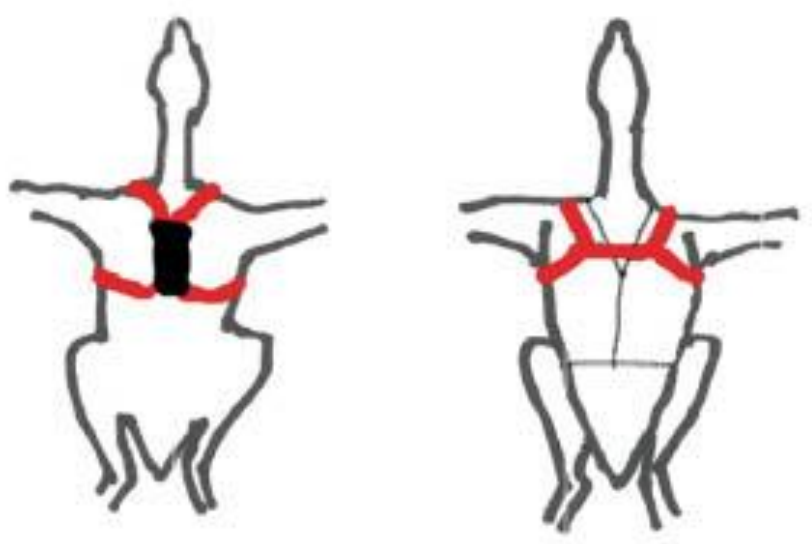

Leg loop
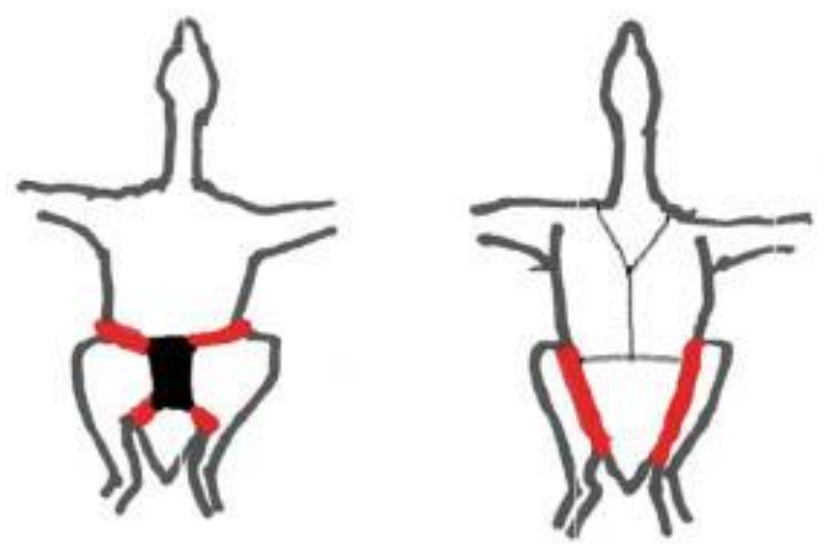


\section{Lateral}

10.

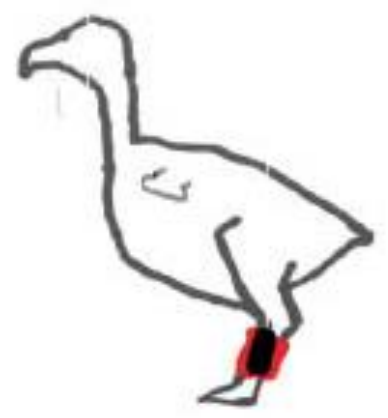

11.

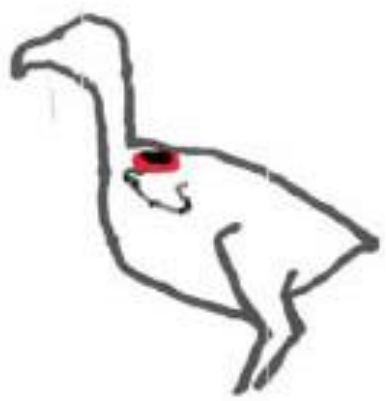

12.

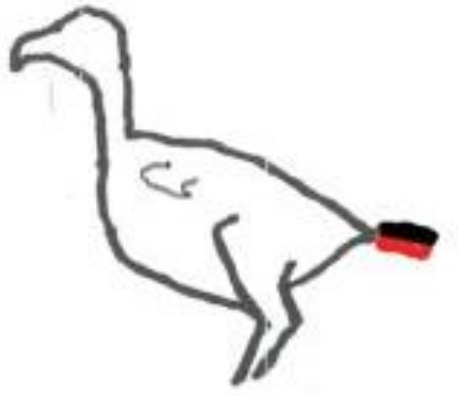

Dorsal

Leg mount
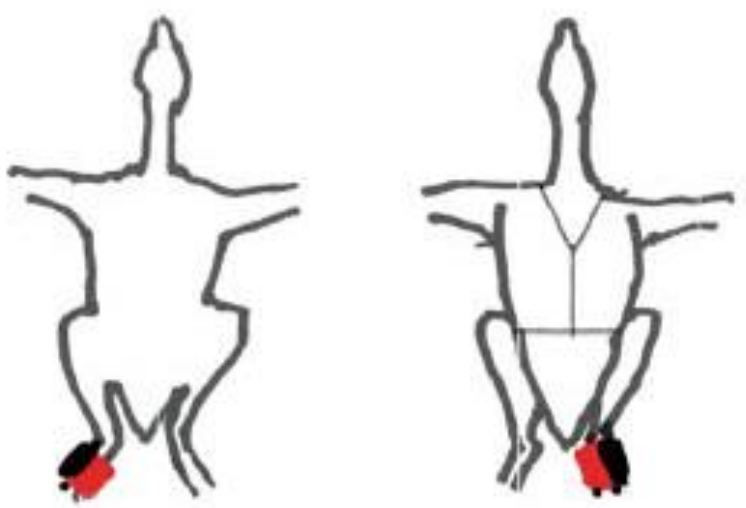

Patagial mount
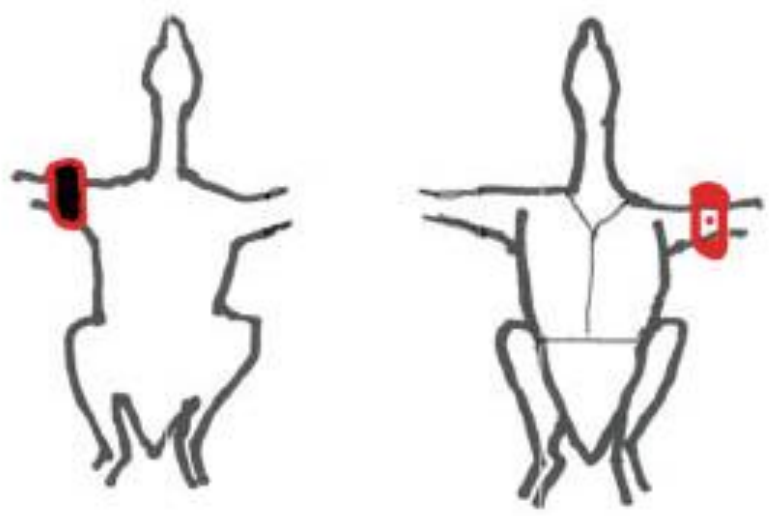

Tail mount
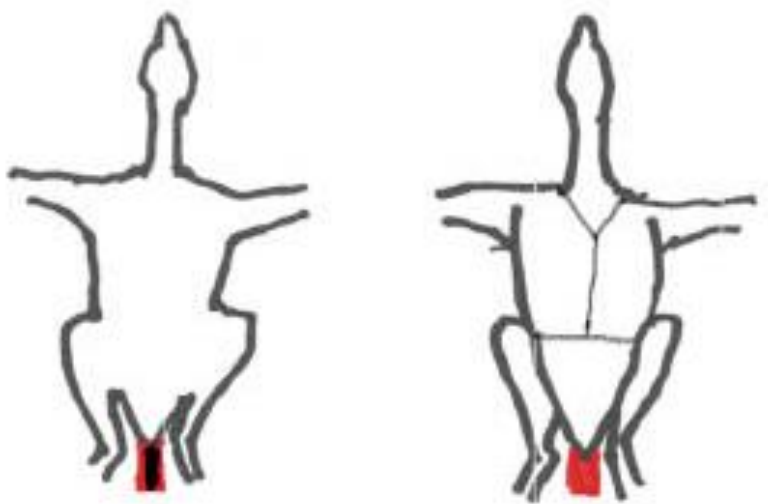

Figure B.1. Diagrams showing 12 designs of attachments used for research devices deployed on Vultures and Condors. 
$* * * * * *$ 